\title{
THE USE OF RESPONSE SURFACE METHODOLOGY FOR RELIABILITY ESTIMATION OF COMPOSITE ENGINEERING STRUCTURES
}

\author{
Karol Winkelmann, Jaroseaw Górski \\ Gdansk University of Technology, Faculty of Civil and Environmental Engineering, Gdańsk, Poland \\ e-mail: karolwin@pg.gda.pl; jgorski@pg.gda.pl
}

\begin{abstract}
Stability loss is one of the key issues in the analysis of shells made of composite materials. This paper aims at finding the variation of the critical or limit value of the load resulting from geometric imperfections. An axially compressed 16-layer cylindrical panel model is considered. The imperfections of the panel are simulated as random fields. The parameters of these fields are used as the basic variables of the reliability problem of the model. The reliability analysis is based on the Response Surface Methodology (RSM). On the basis of the derived equation of the response surface, a reliability index of the model is determined using an author's program - both the Monte Carlo method (MC) and the Hasofer-Lind-Rackwitz-Fiessler (HLRF) reliability index formulation analyses.
\end{abstract}

Keywords: reliability, response surface methodology, composite panel

\section{Introduction}

The key issue in the application of shells made of composite materials is the problem of stability loss, which may be caused by exceeding either the appropriate critical (bifurcation point) or the limit (maximal load point) value of the load.

Variations of the above mentioned load limits can be under strong influence of the appearance of geometric imperfections. This supposition is pointed out in a number of some earlier papers, e.g. Barbero et al., 2000; Rzeszut and Garstecki, 2009).

In this paper, the imperfections are modelled by random fields.

The aim of this study is to investigate the impact of the generated random fields of imperfections on the structural response and to examine the reliability of a shell structure with some random geometric imperfections.

\section{Object of the analysis}

In the investigation, attention is focused on an axially compressed 16-layer cylindrical panel made of carbon-epoxy composite, as shown in Fig. 1. A detailed numerical analysis of the stability loss problem of this shell has been presented earlier, e.g. Kreja (2005), Winkelmann and Sabik (2014).

It is assumed that the straight edges of the panel $(A B$ and $C D)$ are simply supported, and possibility of free movement along the generatrix is taken into account, whereas the curved edges $(B C$ and $D A)$ are clamped. The compression load is realized as a rigid movement of the curved edge $D A$.

The geometry of the panel is characterized by its thickness $t=16 \cdot 0.125 \mathrm{~mm}=2 \mathrm{~mm}$, the radius $R=250 \mathrm{~mm}$, the length $L=540 \mathrm{~mm}$ and the opening angle $\beta=1.6848 \mathrm{rad}$.

The layers of the panel are arranged in accordance with the $\left[45\left|-45_{2}\right| 45 \mid 0_{4}\right]_{S}$ scheme and consist of the XAS-914C composite of the following parameters (per one lamina), $E_{a}=130$. 


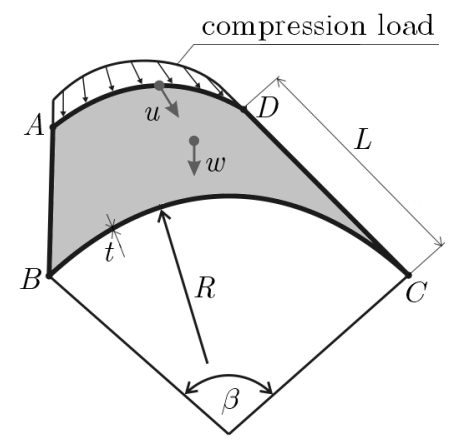

Fig. 1. An axially compressed 16-layer cylindrical panel made of carbon-epoxy composite

$10^{6} \mathrm{kPa}, E_{b}=10 \cdot 10^{6} \mathrm{kPa}, \nu_{a b}=0.3$ and $G_{a b}=G_{a c}=G_{b c}=5 \cdot 10^{6} \mathrm{kPa}$. Taking advantage of the lamination scheme and the parameters of each lamina, a set of overall material parameters of the panel may be calculated by the use of some physical equations for "off-axis" composites, provided by e.g. Kreja (2005), Sabik and Kreja (2011).

The problem is analyzed in commercial software Femap with NX-Nastran (v.10.1.1.). To define the numerical model of the shell, a set of 1600 quadrinodal flat shell elements QUAD4 $(40 \times 40$ elements) is used, spanned on 1681 grid nodes $(41 \times 41$ nodes $)$, given in a polar coordinate system. The definition of the model is based on the large rotations theory, in terms of its co-rotational formulation, utilizing the gimbal angles.

The geometrically and materially nonlinear analysis of the problem is conducted.

\section{Simulation of imperfections using random fields}

In the paper, the geometric imperfections are taken as the only parameter to describe randomness in the shell model. It should be noticed that randomness of the load, boundary conditions, alignment of composite layers and of material parameters are neglected, thus the random description of the model is quite simplified.

The geometric imperfections $w$, which are the input data for a further numerical analysis are modelled as random fields generated by an author's program. A bounded Gaussian distribution is used to generate the random field.

The randomness of the displacements is described by a correlation function for a homogeneous random field, expressed by

$$
K\left(x_{1}, x_{2}\right)=s_{w}^{2} \mathrm{e}^{-\lambda_{x_{1}} \Delta x_{1}}\left(1+\lambda_{x_{1}} \Delta x_{1}\right) \mathrm{e}^{-\lambda_{x 2} \Delta x_{2}}\left(1+\lambda_{x_{2}} \Delta x_{2}\right)
$$

where $\Delta x_{1}$ and $\Delta x_{2}$ are the distances between the points of the random field along the horizontal direction $x_{1}$ and the vertical direction $x_{2}$. The coefficients $\lambda_{x_{1}}$ and $\lambda_{x_{2}}$ are the parameters describing the range of the correlation in both directions, and the standard deviation $s_{w}^{2}$ represents the variability of the field, as suggested in e.g. Bielewicz and Górski (2002), Górski and Mikulski (2008).

In the study, distances $\Delta x_{1}$ and $\Delta x_{2}$ between the points of the field are selected to coincide with the actual dimensions of the panel. An assumption is made that the standard deviation of the field is unitary $\left(s_{w}^{2}=1\right)$. The coefficients $\lambda_{x_{1}}$ and $\lambda_{x_{2}}$ have no numerical values. However, they are represented by two identically distributed random variables $\lambda$, defined on a sample space $\mathbf{x}$.

In order to generate the random fields modelling the geometric imperfections by applying the above mentioned correlation function (3.1), the conditional acceptance and rejection generation method is used. This method is described in detail in e.g. Bielewicz and Górski (2002). 
The application of the Finite Element Method to deal with this problem requires averaging of the values of the generated random field of imperfections. The procedure proposed in Vanmarcke (1983) is used for this purpose, as can be seen in equations (3.2)

$$
\begin{aligned}
& D_{w}(\Delta x, \Delta y)=\frac{2}{\lambda_{x} \Delta x}\left[2+\mathrm{e}^{-\lambda_{x} \Delta x}-\frac{3}{\lambda_{x} \Delta x}\left(1-\mathrm{e}^{-\lambda_{x} \Delta x}\right)\right] \\
& \cdot \frac{2}{\lambda_{y} \Delta y}\left[2+\mathrm{e}^{-\lambda_{y} \Delta y}-\frac{3}{\lambda_{y} \Delta y}\left(1-\mathrm{e}^{-\lambda_{y} \Delta y}\right)\right] \\
& K_{w}(\Delta x, \Delta y)=\frac{\mathrm{e}^{\lambda_{x} \Delta x}}{\left(\lambda_{x} \Delta x\right)^{2}}\left\{\left[\cos \left(\lambda_{x} \Delta x\right)-\sin \left(\lambda_{x} \Delta x\right)\right]+2 \lambda_{x} \Delta x-1\right\} \\
& \cdot \frac{\mathrm{e}^{\lambda_{y} \Delta y}}{\left(\lambda_{y} \Delta y\right)^{2}}\left\{\left[\cos \left(\lambda_{y} \Delta y\right)-\sin \left(\lambda_{y} \Delta y\right)\right]+2 \lambda_{y} \Delta y-1\right\}
\end{aligned}
$$

The generated random field is implemented in Femap using the NX-Nastran numerical model of the shell as a map of the geometric imperfections described in form of a disturbance of the mesh of the model introduced before generating the shell elements.

\section{Adoption of random variables}

A plot of the equilibrium path of the axial displacement $U$, shown illustratively in Fig. 1, is traced as a result of a single series of calculations. Two specific points are considered along this path. The first one is the displacement of the central node of the curved edge $U_{101}$ for a loading level of $80 \mathrm{kN}$ (the reference level that ensures stability for every path under consideration), while the second one is the stability loss point, in which the appropriate critical or limit value of the load is reached.

The second-level reliability estimate is made for the present numerical model by means of the Response Surface Methodology. A study of current development and computational competitiveness of RSM is presented in e.g. Gomes and Awruch (2004), Chiachio et al. (2012).

Apart from the choice of methodology, the problem of proper and justified (from an engineering point of view) selection of random variables for the task and a proper assessment of their moments is of great importance. Special attention should be paid to the choice of the starting point of the analysis (zero values for all variables). All the reliability indices are calculated with respect to that point (see e.g. Kamiński, 2005; Postek et al., 2002).

On the basis of some earlier studies related to the subject and some preliminary numerical tests (carried out for regular fields of imperfections, e.g. for a single paraboloid, two parabolas stretched along the entire length of both edges of the panel and for an uncorrelated random field) it is noted that the greatest impact on the variation of the load value at which the stability loss of the panel occurs is caused by only two factors (see e.g. Winkelmann and Sabik, 2014).

The first factor is the imperfection size, taken as a random variable of the task. In the case of the random fields, the amplitude of the field may be equivalently assumed.

However, it should be noted that the first random variable $x_{1}$ (the amplitude variable $m=m_{x_{1}}=m_{x_{2}}$ ) is determined from a uniform distribution in the range of $x_{1} \in\langle 0.0 ; 1.0\rangle$, where the numerical value of the variable taken from the range represents a reducing multiplier applied to the normalized random field.

By the use of some earlier numerical tests, the generated random field amplitude is converted into a random variable value. First, it is assumed that each generated random field is provided with a subsequent normalization, as mentioned before. In the process, the field gains only one point of the maximum amplitude being always equal to unity. The obtained unitary value is expressed in centimeters. 
This ensures that the point of the maximum displacement of the field is, in fact, generated by a random value between $0 \mathrm{~cm}$ (shell with a perfect geometry, $x_{1}=0.0$ ) and $2 \mathrm{~cm}$ (shell with the highest amplitude of the field of imperfections, the maximum deviation from the ideal geometry, $\left.x_{1}=1.0\right)$.

The value of the parameters describing the correlation extent in both directions is decided to be the second random variable of the task. Due to the attempts to limit the complexity of the analysis, and to present the results in three-dimensional graphical form, the parameters have been assumed to create only one random variable, of the same value for both the horizontal and vertical dimensions. Making use of the previous numerical tests, it has been taken for granted that the second random variable $x_{2}$ (the correlation variable $\lambda=\lambda_{x_{1}}=\lambda_{x_{2}}$ ) is also chosen from a uniform distribution in the range of $x_{2} \in\langle 0.0 ; 1.0\rangle$ with its numerical value taken from the range representing directly the value of the correlation extent parameter.

On the assumption that there is a normalized random field, as done in this analysis, the impact of the correlation extent parameter on the shape of a random field can be indicated. Figure 2 shows the impact of the correlation factor (as shown on the vertical axis) on a specified number of finite elements of the considered composite panel (as shown on the horizontal axis), in relation to the correlation extent parameter $\lambda$ adapted in the process of random fields generation.

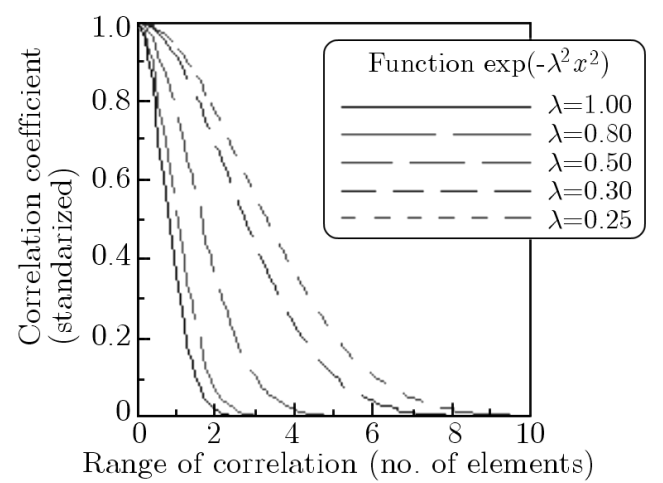

Fig. 2. Influence of the correlation factor on the specified number of finite elements of the considered composite panel in relation to the correlation extent parameter $\lambda$
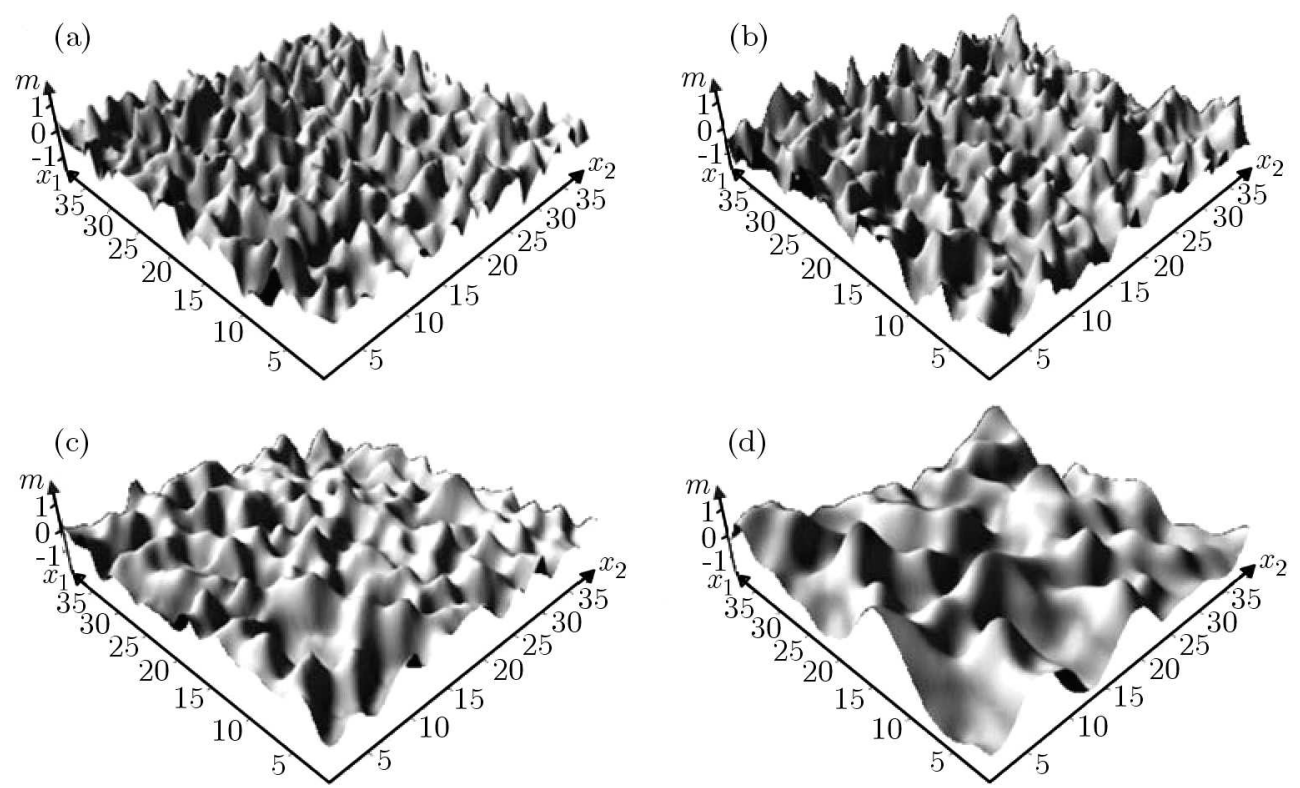

Fig. 3. Surfaces of arbitrarily generated random fields with different values of the correlation extent parameter $\lambda$ : (a) random field of very weak correlation, $\lambda=\lambda_{x_{1}}=\lambda_{x_{2}}=1.00$; (b) random field of weak correlation, $\lambda=\lambda_{x_{1}}=\lambda_{x_{2}}=0.80$; (c) random field of medium correlation, $\lambda=\lambda_{x_{1}}=\lambda_{x_{2}}=0.50$;

(d) random field of strong correlation, $\lambda=\lambda_{x_{1}}=\lambda_{x_{2}}=0.30$ 
Figure 3 presents a number of surfaces of arbitrarily generated random fields with different values of the correlation extent parameter $\lambda$.

\section{Numerical calculations}

Following the input data used for the analysis described above, a series of numerical calculations is conducted, leading to the determination of the equilibrium path of the axial displacement $U$ of the loaded shell edge. A series of 24 calculations is carried out in three stages adjusted to a later application of the response surface methodology. Every calculation is recorded with respect to the displacement of the central node of the curved edge $U_{101}$ for a loading level of $80 \mathrm{kN}$ and a load value resulting in the loss of stability.

In the first stage, 6 samples are analyzed in order to perform the first approximation of the response surface. Therefore, four sampling points are selected from the area close to the corners of the approximated surface with the $(m ; \lambda)$ coordinates as follows: $(0.01 ; 0.30),(0.01 ; 0.80)$, $(1.00 ; 0.30)$ and $(1.00 ; 0.80)$ to correctly distribute the surface in the sample space. Two more points are selected near the center of the approximated surface with the $(m ; \lambda)$ coordinates of: $(0.05 ; 0.50)$ and $(0.10 ; 0.50)$ to approximate the convexity of the surface in the middle of its span.

In the second stage, the next 6 samples are analyzed. They are chosen to collect information about the response surface near its edges. The selected points have the following $(m ; \lambda)$ coordinates $(0.05 ; 0.30),(0.10 ; 0.30),(0.05 ; 0.80),(0.10 ; 0.80),(0.01 ; 0.50)$ and $(1.00 ; 0.50)$.

In the third stage, further 12 samples are subjected to analysis. This time, they are chosen completely at random (using the direct Monte Carlo sampling), just to specify the information relating to the response surface near its center. The selected points have the following $(m ; \lambda)$ coordinates $(0.43 ; 0.74),(0.38 ; 0.75),(0.75 ; 0.42),(0.66 ; 0.71),(0.69 ; 0.39),(0.23 ; 0.67),(0.30 ; 0.38)$, $(0.52 ; 0.43),(0.84 ; 0.72),(0.85 ; 0.53),(0.21 ; 0.56)$ and $(0.12 ; 0.44)$.

The equilibrium paths for the axial displacement $U$ obtained in Femap using the NX Nastran calculation software regarding all the sampling points of the analysis are presented in Fig. 4. It should be noted, on the basis of the figure attached, that for the load level equal to $80 \mathrm{kN}$ the stability in every path under consideration is completely ensured.

Prior to the response surface approximation procedures and the assessment of reliability of the panel, the limit state boundary conditions relating to all cases of shell imperfections should be formulated.

It is assumed in the problem that in the first case of the analysis the displacement of the central node of the curved edge $U_{101}$ for a loading level of $80 \mathrm{kN}$ should not exceed the limit value of $U_{101, \text { lim }}=2.0 \mathrm{~mm}$, which is equal to the thickness of the entire composite element. In the second case of the analysis - the point of stability loss, at which the corresponding critical or limit load value is reached, should not be placed on the equilibrium path lower than the level of $100 \mathrm{kN}$.

\section{Convergence analysis of the obtained results}

While assessing the accuracy of estimation of probability of failure of composite panels using the direct Monte Carlo method, one of the key issues is to observe the convergence relating to the results of the probabilistic moments of the structural response due to an increment in the sample population. Therefore, a simple convergence analysis of both the cases of the considered task is performed. 
(a)

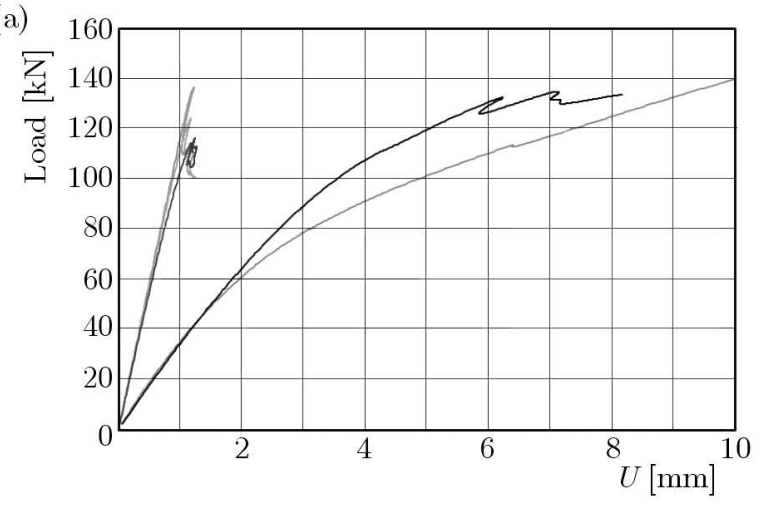

(b)

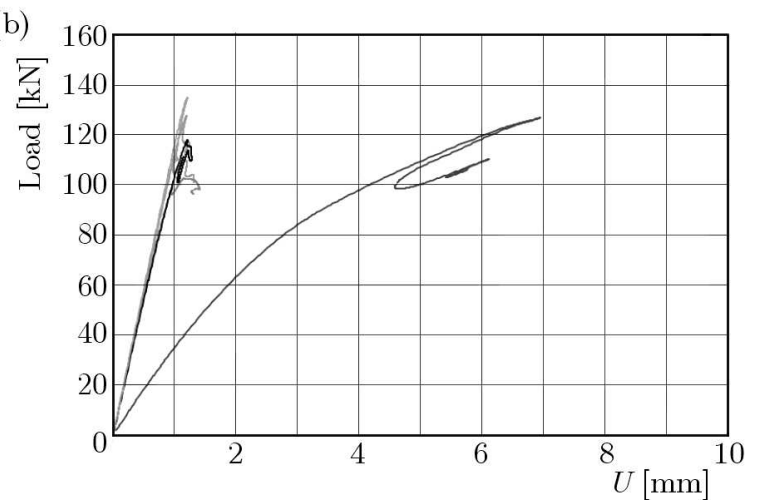

(c)

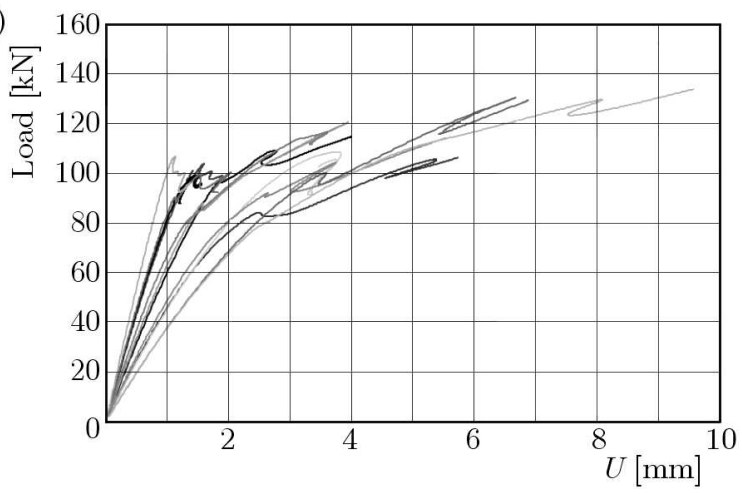

Fig. 4. The equilibrium paths for the axial displacement $U$ obtained in Femap using the NX Nastran software for the geometrically imperfect shell model: (a) first stage of analysis (6 samples), (b) second stage of analysis (6 samples), (c) third stage of analysis (12 samples)

\subsection{Displacement of the central node of the curved edge $U_{101}$ for a loading level of $80 \mathrm{kN}$}

Convergence of the results of the probabilistic moments of the structural response (probability of failure) when tracking the displacement of the central node of the curved edge for a loading level of $80 \mathrm{kN}$ is shown in Fig. 5 .

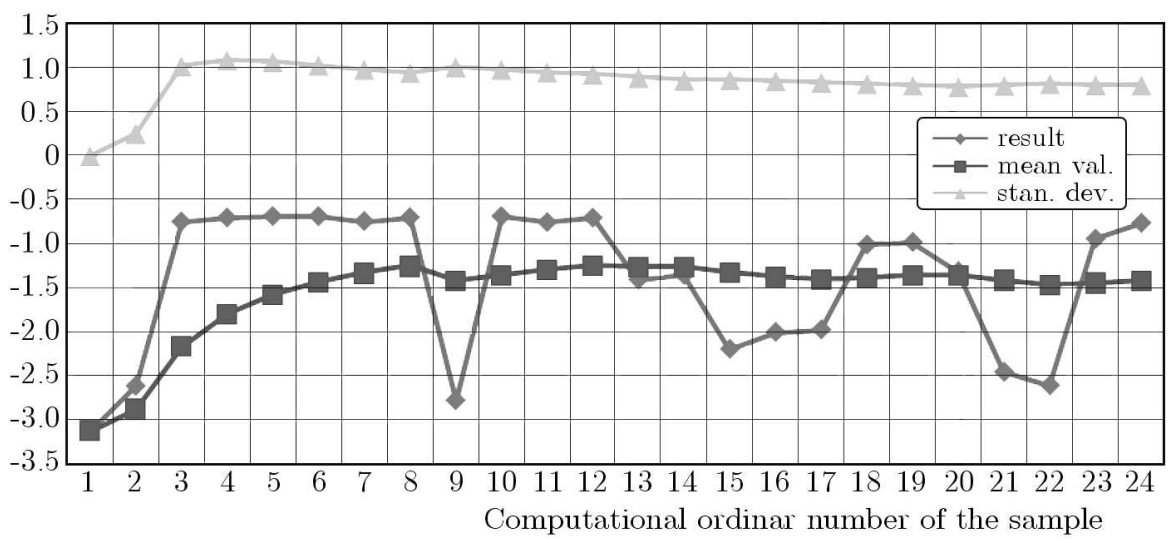

Fig. 5. Convergence of the probabilistic moments of the structural response (probability of failure) when tracking the displacement of the central node of the curved edge for a loading level of $80 \mathrm{kN}$

Figure 5 proves that the first stage of the analysis (including only 6 samples) does not imply a satisfactory convergence. This is surely connected with a significant divergence of the displacements observed at the midpoints and in the corners of the approximated surface. It is a very interesting phenomenon since it indicates that the samples of an average correlation and a relatively small amplitude have a much smaller inclination to their equilibrium paths with 
respect to the axis of the $U_{101}$ displacement than the equilibrium paths in the samples with a small or strong correlation and a significant amplitude. This may be associated with the fact that a large amplitude of the field of imperfections results in a completely different geometry of the shell, behaving like a corrugated object, not a flat one.

Due to the use of additional samples in the second stage of analysis, the observed convergence after examining 12 samples is satisfactory. The difference noted in the two final results of the average displacement $U_{101}$ is only $3.80 \%$. However, in consequence of the arrangement of samples along the response surface, it is difficult to say that this choice corresponds closely to the assumptions of the Monte Carlo methodology. The consideration of the consecutive samples, selected from the variation ranges of the variables in a strictly random way, therefore makes it necessary to prove the applicability of the Monte Carlo method to expected structural failures.

On the basis of the third stage of analysis (including 24 samples), the convergence of probabilistic moments of probability of failure is almost complete. The difference between two final results of the average displacement $U_{101}$ in the third stage of the analysis is only $2.01 \%$, whereas the analogous difference of standard deviations of the $U_{101}$ displacement reaches only $0.68 \%$. Both the differences prove the convergence of calculations, and thus their results may be worthy of attention. This statement is not affected by a significant scatter of the displacement values relating to a loading level of $80 \mathrm{kN}$, introduced by a diversity of localization of the calculation points (samples) in the third stage of the procedure.

Such a situation proves that the estimation of the structural probability of failure using the Monte Carlo method provided with a small number of calculation points (samples) is evidently possible, even in complex engineering structures, if only the distribution of the samples are chosen in an appropriate manner.

\subsection{Load value resulting in stability loss}

Convergence of the probabilistic moments of the structural response (probability of failure) when tracking the load values causing stability loss is shown in Fig. 6.

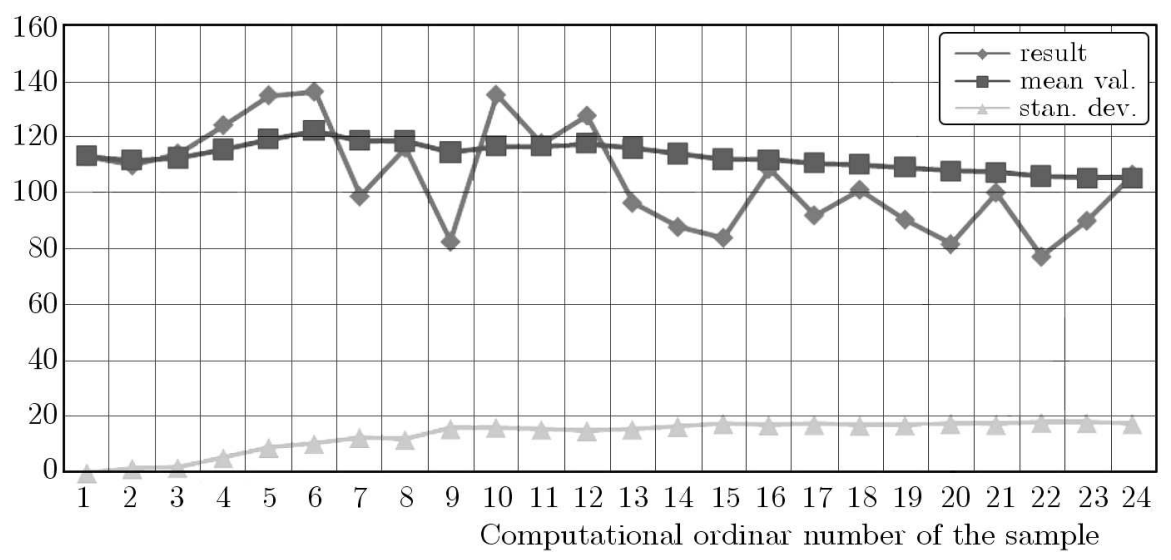

Fig. 6. Convergence of the probabilistic moments of the structural response (probability of failure) when tracking the load values causing stability loss

Figure 6 proves that following the first stage of the analysis (with only 6 samples), a satisfactory convergence has not been reached in this example either. Yet, after the second stage of study (involving a total of 12 samples), a convergence of the mean value of the load resulting in the stability loss and its standard deviation improved significantly. However, it should be emphasized that the calculation points generated by the Monte Carlo method have not been included in the analysis yet, thus the choice of the points is not a part of a crude Monte Carlo sampling procedure. 
In the results obtained from the above mentioned calculation points at the end of the third stage (the analysis of a total of 24 samples), it may be noted that the convergence of the mean value of the load resulting in the stability loss is adjusted to a value slightly different (a difference of $10.4 \%$ ) from the value of the second stage. This calls for necessary analysis of the most varied range of the calculation points. Convergence of the standard deviation of the load resulting in the stability loss remains unchanged, like at the end of the previous two stages (just as in the analysis of the previous case), which also supports the choice of the 24 calculation points (samples) in a manner described above.

\section{The response surface approximation results}

A competitive reliability analysis is provided using the Response Surface Methodology (RSM), together with a tabular Analysis of Variance procedure, described in detail by Rajashekhar and Ellingwood (1993).

Assuming that the curvature of the approximated response surface is large, but not very complicated, a second-order approximation model with cross-terms is used.

Its form, as used in this task (with only two random variables) is expressed by

$$
\widehat{g}(\mathbf{x})=\beta_{0}+\beta_{1} x_{1}+\beta_{2} x_{2}+\beta_{11} x_{1}^{2}+\beta_{22} x_{2}^{2}+\beta_{12} x_{1} x_{2}
$$

An assumption is made that the response surface approximation is conducted in three stages, like in the Monte Carlo method reliability estimation, taking into consideration the sequence of 6, 12 and 24 previously mentioned calculation points.

The approximation of the response surface is performed individually not only with regard to every stage of a single variant of the task, but also both the previously mentioned variants.

\subsection{Displacement of the central node of the curved edge $U_{101}$ for a loading level of $80 \mathrm{kN}$}

Equation (7.2) illustrates the response surface obtained for 6 calculation points

$$
\widehat{g}(\mathbf{x})=1.268+0.267 x_{1}-1.197 x_{2}-0.253 x_{1}^{2}-1.558 x_{2}^{2}+1.033 x_{1} x_{2}
$$

An illustrative diagram of the expected structural response surface approximated by using 6 calculation points (samples) is shown in Fig. 7a.

Equation (7.3) illustrates the response surface obtained for 12 calculation points

$$
\widehat{g}(\mathbf{x})=1.200+0.598 x_{1}-1.061 x_{2}-0.594 x_{1}^{2}-1.653 x_{2}^{2}+1.044 x_{1} x_{2}
$$

An illustrative diagram of the expected structural response surface approximated by using 12 calculation points (samples) is shown in Fig. 7b.

Equation (7.4) illustrates the response surface obtained for 24 calculation points

$$
\widehat{g}(\mathbf{x})=1.447-0.061 x_{1}-1.634 x_{2}-0.145 x_{1}^{2}-0.990 x_{2}^{2}+0.674 x_{1} x_{2}
$$

A similar diagram of the expected structural response surface approximated by using 24 calculation points (samples) is shown in Fig. 7c.

\subsection{Load value resulting in stability loss}

Equation (7.5) illustrates the response surface obtained for 6 calculation points

$$
\widehat{g}(\mathbf{x})=46.16-42.80 x_{1}-225.34 x_{2}+41.44 x_{1}^{2}+204.20 x_{2}^{2}-9.30 x_{1} x_{2}
$$





Fig. 7. Illustrative diagrams of the predicted structural response surfaces obtained by approximation of: (a) 6, (b) 12 and (c) 24 calculation points

An illustrative diagram of the predicted structural response surface approximated using 6 calculation points (samples) is shown in Fig. 8a.

Equation (7.6) illustrates the response surface obtained for 12 calculation points

$$
\widehat{g}(\mathbf{x})=47.46-64.41 x_{1}-298.28 x_{2}+76.17 x_{1}^{2}+271.69 x_{2}^{2}-17.81 x_{1} x_{2}
$$

An illustrative diagram of the predicted structural response surface approximated using 12 calculation points (samples) is shown in Fig. 8b.

Equation (7.7) illustrates the response surface obtained for 24 calculation points

$$
\widehat{g}(\mathbf{x})=51.19-118.46 x_{1}-134.89 x_{2}+124.71 x_{1}^{2}+111.41 x_{2}^{2}-2.05 x_{1} x_{2}
$$

An illustrative diagram of the predicted structural response surface approximated using 24 calculation points (samples) is shown in Fig. 8c.

Making use of the plotted response surfaces in both cases of the analysis, it can be clearly stated that the proposed initial stages under investigation (for 6 and 12 calculation points used to predict the surfaces, for which the calculation samples are grouped mostly on the edges of the considered segment of the surface), depict the global behaviour of the response surfaces in a satisfactory manner. However, for a full recognition of the response surface topography it is necessary to add a certain amount of calculation samples to significantly improve the quality of the approximation. As for this task, only 12 points are added. It indicates that the number of some extra samples can be smaller if they are selected in an appropriate manner.

It is worth mentioning that in the first case of the analysis, in the two starting stages, a correct topography of the response surface is found, but its elevation above the limit state equation reference level is set incorrectly. In the second case of the analysis, in the two starting stages, a proper elevation of the response surface above the reference level is found, but its topography is rated erroneously (a "saddle" is shown, instead of a "funnel"). Both of the above 

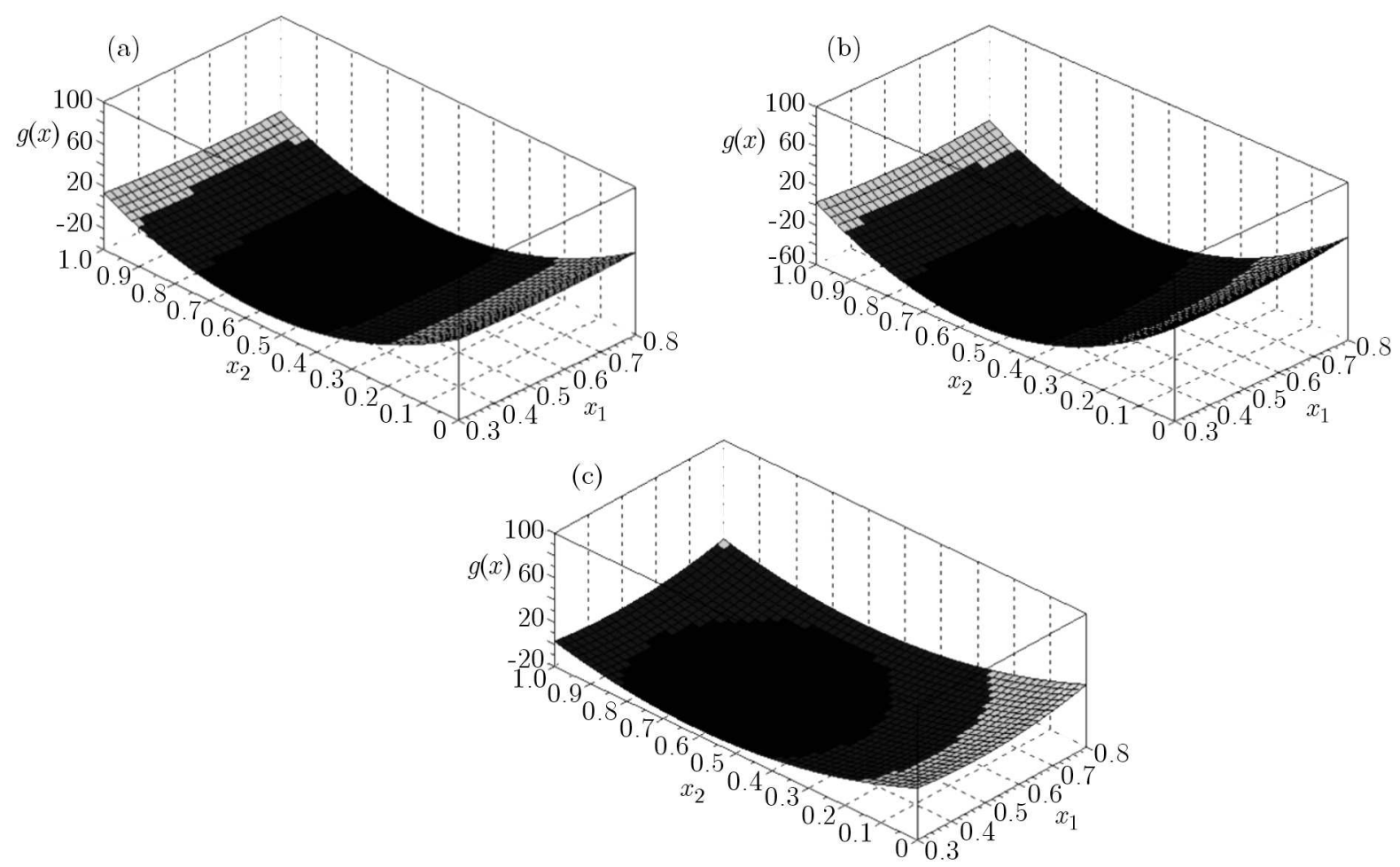

Fig. 8. Illustrative diagrams of the predicted structural response surfaces obtained by approximation of: (a) 6, (b) 12 and (c) 24 calculation points

mentioned deficiencies could lead to a wrong probabilistic assessment of structural safety. Thus, the calculations should be finished only after considering a sufficient number of calculation points.

The total of 24 calculation points appears to be sufficient for such a complex engineering task. It is expected that the results obtained after the third stage of both the calculations (including two cases of the analysis) are of decisive significance relating not only to probability of failure but also to the reliability indices, the Hasofer-Lind index $\beta_{H L}$ and the Hasofer-Lind-Rackwitz-Fiessler index $\beta_{H L R F}$.

\section{Analysis of probability of shell failure}

It is assumed that nine different parameters of the probabilistic reliability assessment are examined in the analysis of the considered composite panel.

These parameters are calculated in the following order:

- the probability of failure of the panel calculated using the direct Monte Carlo approach $P_{f, M C}$ as a ratio of the number of samples satisfying the limit state equation to the total amount of the sample space;

- the safety of the panel calculated by the application of the direct Monte Carlo approach $P_{s, M C}$ as opposed to the above defined probability of failure given by $P_{s, M C}=$ $1-P_{f, M C}$

- Cornell's reliability index calculated by the use of the direct Monte Carlo approach $\beta_{M C}$, defined as $\beta_{M C}=-\Phi^{-1}\left(P_{f}\right)$;

- Hasofer-Lind reliability index $\beta_{H L, R S M}$ calculated on the basis of the approximated response surface equation; 
- the corresponding probability of failure of the panel, given by $P_{f, H L, R S M}=\Phi\left(-\beta_{H L, R S M}\right)$;

- the corresponding safety of the panel, expressed by $P_{s, H L, R S M}=1-P_{f, H L, R S M}$;

- Hasofer-Lind-Rackwitz-Fiessler reliability index $\beta_{H L R F, R S M}$, also calculated on the basis of the approximated response surface equation;

- the corresponding probability of failure of the panel, given by $P_{f, H L R F, R S M}=$ $\Phi\left(-\beta_{H L R F, R S M}\right)$;

- the corresponding safety of the panel, given in form of $P_{s, H L R F, R S M}=1-P_{f, H L R F, R S M}$.

In the numerical calculations performed by means of the author's program, it is assumed that the calculated values of the reliability indices are proper after twenty iterations. The program is described in Winkelmann (2014).

All parameters of the probabilistic reliability assessment of the shell are calculated with the data taken from all three calculation stages (including 6, 12 and 24 calculation points) individually.

The estimates are made in separate operations using both the criteria for the task, i.e. the displacement of the central node of the curved edge $U_{101}$ for a loading level of $80 \mathrm{kN}$, and a load value leading to stability loss.

The results of the probabilistic parameters in the reliability assessment, for a successively increasing number of the samples for the first variant of the task when tracking the displacement of the central node of the curved edge for a loading level of $80 \mathrm{kN}$, are presented in Table 1 .

Table 1. A set of results of the parameters of probabilistic safety for the first variant of the task - the tracking of the displacement of the central node of the curved edge for a loading level of $80 \mathrm{kN}$

\begin{tabular}{|c|c|c|c|}
\hline $\begin{array}{c}U_{101} \\
\text { at } 80 \mathrm{kN}\end{array}$ & Stage 1 & Stage 2 & Stage 3 \\
\cline { 2 - 4 } & 6 samples & 12 samples & 24 samples \\
\hline \hline$P_{f, M C}$ & 0.333 & 0.250 & 0.250 \\
\hline$P_{s, M C}$ & 0.667 & 0.750 & 0.750 \\
\hline$\beta_{M C}$ & 0.430 & 0.675 & 0.675 \\
\hline$P_{f, H L, R S M}$ & 0.217 & 0.197 & 0.250 \\
\hline$P_{s, H L, R S M}$ & 0.783 & 0.803 & 0.750 \\
\hline$\beta_{H L, R S M}$ & 0.783 & 0.853 & 0.673 \\
\hline$P_{f, H L R F, R S M}$ & 0.191 & 0.142 & 0.176 \\
\hline$P_{s, H L R F, R S M}$ & 0.809 & 0.858 & 0.824 \\
\hline$\beta_{H L R F, R S M}$ & 0.875 & 1.076 & 0.932 \\
\hline
\end{tabular}

Analogous results for the second variant of the task, in consequence of tracking the load values resulting in the stability loss, are presented in Table 2.

Based on the data presented in Table 1 and 2, analysis of convergence of the parameters of probabilistic reliability estimation is performed (separately for each variant of the task) in all three successive stages of both the analyses.

The first variant of the task is to examine if the displacement of the central node of the curved edge $U_{101}$ for a loading level of $80 \mathrm{kN}$ exceeds the limit value of $2 \mathrm{~mm}$. The calculations of the probabilistic parameters in the reliability assessment of the shell (both the probability of failure of the panel $P_{f}$ and the reliability indices $\beta$ ) are completed after the three stages of computations (following the calculations of the response surface involving all 24 calculation points) with an insufficient convergence of the proposed solutions.

When analyzing the two stages with 12 and 24 sample points, a significant reduction is detected in the position of the response surface with respect to the axis of the limit state 
Table 2. A set of results of the parameters of probabilistic safety for the second variant of the task - the tracking of the load values resulting in the stability loss

\begin{tabular}{|c|c|c|c|}
\hline \multirow{2}{*}{$N_{C R I T}$} & Stage 1 & Stage 2 & Stage 3 \\
\cline { 2 - 4 } & 6 samples & 12 samples & 24 samples \\
\hline \hline$P_{f, M C}$ & 0.000 & 0.167 & 0.417 \\
\hline$P_{s, M C}$ & 1.000 & 0.833 & 0.583 \\
\hline$\beta_{M C}$ & 3.999 & 0.970 & 0.210 \\
\hline$P_{f, H L, R S M}$ & 0.265 & 0.327 & 0.381 \\
\hline$P_{s, H L, R S M}$ & 0.735 & 0.673 & 0.619 \\
\hline$\beta_{H L, R S M}$ & 0.627 & 0.447 & 0.304 \\
\hline$P_{f, H L R F, R S M}$ & 0.374 & 0.394 & 0.406 \\
\hline$P_{s, H L R F, R S M}$ & 0.626 & 0.606 & 0.594 \\
\hline$\beta_{H L R F, R S M}$ & 0.320 & 0.270 & 0.139 \\
\hline
\end{tabular}

function $\widehat{g}(\mathbf{x})$ for the random variables $x_{1}, x_{2}$. This makes the reliability indices calculated by using the surface change their values almost parallel to each other between the second and third stage, instead of being convergent (consistently, the corresponding probability of failure reacts the same). However, the probabilistic parameters of the shell reliability assessment given by the Monte Carlo method are characterized by a high rate of convergence as a result of applying the RSM-based Hasofer-Lind approach. Therefore, it can be expected that in the case of the determination of the response surface localization level in the space of random variables in the subsequent series of calculation performed using more than 24 samples, these results will have a more satisfactory convergence.

The second variant of the task aims at finding out if the point of stability loss in which the corresponding critical or limit value of the load is reached, is situated on the equilibrium path lower than the level of $100 \mathrm{kN}$. This case of the assessment of probabilistic parameters of the shell reliability is completed after three stages of computations with an almost perfect convergence.

Taking into consideration the two stages with 12 and 24 sample points, the recognition of the surface topography has improved significantly, the limit state boundary is smoothened and the numerical approximation is better. This is noted in form of rapid and satisfactory convergence of the assessment of the probabilistic parameters of the shell reliability calculated on the basis of the RSM. The reference results of the Monte Carlo simulations have the same satisfactory convergence, after the third stage of the analysis, as the results obtained from the author's program.

The presented results confirm that a small number of calculation points selected at random may quickly lead to good solutions of the reliability indices. The lack of convergence of stage 2 and 3 in the first variant of the analysis could probably be avoided if the order of the calculation points is mixed (the first two stages model only the verges of the surface, whereas the third one models its middle part - such a sequence of operation has proved to be effective, but not optimal).

However, on the basis of the reported results, an extensive analysis of such problems is highly recommended. To deal with such an advanced engineering problem, the probabilistic reliability assessment cannot be reduced to the calculation of only one arbitrarily chosen reliability index. The presented final value of the index should be verified with another approach (e.g. the Monte Carlo method), which is not only a confirmation of the reliability parameters, but also, more importantly, a measure of the correctness of the response surface approximation and its topography recognition, as can be seen in the second variant of the analysis. 


\section{Summary}

In the considered problem, the Response Surface Methodology (RSM), together with a tabular Analysis of Variance procedure is efficiently used to provide the response surfaces of an imperfect composite shell, for which the imperfection parameters are modelled as random variables. Using two mathematical approaches, two reliability indices are calculated for the shell. An advantage is successfully taken of an author's program in that process. In addition, the probability of failure and the subsequent reliability index are also estimated using the Monte Carlo method to verify the previously mentioned indices computed by means of the response surfaces.

On the basis of the undertaken task, it has been proved that the calculations carried out by the use of the random character of the composite shell model (in this case - the assumption of the random field to model the geometric imperfections distributed on the surface of the panel) are fully justified and may also be indicated in engineering design calculations. Thus it is possible to estimate the reliability of this type of structures and to formulate a method to calculate the reliability for geometrically nonlinear problems in a wider range than these presented in this paper.

Another important fact is clearly noted while performing the calculations. The geometric imperfections that may really occur in such dimensions and configurations in some types of shell structures apparently differentiate their structural responses (mostly their capacity and serviceability).

Acknowledgements

This contribution has been supported by the National Science Centre under grant No. UMO-2011/03/B/ST8/06500.

\section{References}

1. Barbero E., Fernandez-Saez J., Navarro C., 2000, Statistical analysis of the mechanical properties of composite materials, Composites, Part B: Engineering, 31, 2, 375-381

2. Bielewicz E., Górski J., 2002, Shell with random geometric imperfections - imulation-based approach, International Journal of Non-linear Mechanics, 37, 4-5, 777-784

3. Chiachio M., Chiachio J., Rus. G., 2012, Reliability in composites - a selective review and survey of current development, Composites: Part B: Engineering, 43, 3, 902-913

4. Gomes H. M., Awruch A. M., 2004, Comparison of response surface and neural network with other methods for structural reliability analysis, Structural Safety, 26, 49-67

5. Górski J., Mikulski T., 2008, Identification and simulation of initial geometric imperfections of steel cylindrical tanks, Journal of Theoretical and Applied Mechanics, 46, 2, 413-434

6. Kamiński M.M., 2005, Computational Mechanics of Composite Materials: Sensitivity, Randomness and Multiscale Behaviour, Springer-Verlag, London

7. Kreja I., 2005, Stability analysis of composite cylindrical panels in the NASTRAN system, Archives of Civil and Mechanical Engineering, 5, 31-41

8. Postek E., Siemaszko A., Kleiber M., 2002, Reliability study of a containment shell, Journal of Theoretical and Applied Mechanics, 40, 1, 179-203

9. Rajashekhar M.R., Ellingwood B., 1993, A new look at the response surface applications for reliability analysis, Structural Safety, 12, 205-220

10. Rzeszut K., Garstecki A., 2009, Modelling of initial geometric imperfections in stability analysis of thin - walled structures, Journal of Theoretical and Applied Mechanics, 47, 3, 667-684 
11. SABiK A., Kreja I., 2011, Stability analysis of multilayered composite shells with cut-outs, Archives of Civil and Mechanical Engineering, 11, 195-207

12. Vanmarcke E.-H., 1983, Random Fields: Analysis and Synthesis, MIT Press, Cambridge

13. Winkelmann K., 2014, The use of Response Surface Methodology for reliability estimation of aluminum silo subjected to wind load, Shell Structures: Theory and Applications, 3, 571-574

14. Winkelmann K., SABiK A., 2014, Computations of critical load value of composite shell with random geometric imperfections, Shell Structures: Theory and Applications, 3, 267-270

Manuscript received September 26, 2013; accepted for print February 2, 2014 